\title{
L-band Digital Aeronautical Communications System (LDACS) - Technical Validations in SESAR2020
}

\author{
Christoph Rihacek \\ Corporate Research \\ Frequentis $A G$ \\ Vienna, Austria \\ christoph.rihacek@frequentis.com \\ Josef Meser \\ Corporate Research \\ Frequentis $A G$ \\ Vienna, Austria \\ josef.meser@frequentis.com
}

\author{
Miodrag Sajatovic \\ Corporate Research \\ Frequentis $A G$ \\ Vienna, Austria \\ miodrag.sajatovic@frequentis.com \\ Thomas Gräupl \\ Institute of Communications and Navigation \\ German Aerospace Center (DLR) \\ Wessling, Germany \\ thomas.graeupl@dlr.de
}

\begin{abstract}
Long-term air traffic management (ATM) concepts for Europe and other regions with rapidly increasing number of aircraft movements require modern and performant mobile data links for safety-related air-ground communications. L-band Digital Aeronautical Communications System (LDACS) is one of the new technologies, which is tailored to the specific needs of the ATM community and developed in the Single European Sky ATM Research (SESAR) program. This paper illustrates results of the technical validations of LDACS carried-out in the SESAR2020 project "PJ.14 0201 Future Communication Infrastructure (FCI) Terrestrial Data Link", focusing on aspects related to LDACS physical layer and data link layer.
\end{abstract}

\section{Keywords—SJU, LDACS, mobility management.}

\section{INTRODUCTION}

An essential enabler for sustainable air traffic management (ATM) systems is a modern communication infrastructure that enables efficient aircraft control and safe separation in all phases of flight. Current ATM communications systems are mature, but they suffer from VHF band's increasing saturation in areas with high flight traffic density. Therefore, the European Union strives for modernization of the aeronautical communication infrastructure to increase airspace capacity.

In the long-term, ATM communication shall migrate from analogue VHF voice communication to more spectrum efficient digital data communication. The European ATM Master Plan [1] foresees this transition to be realized, among others, through the development and implementation of the L-band Digital Aeronautical Communications System (LDACS).

LDACS development has already made substantial progress in the Single European Sky ATM Research (SESAR) framework and it is currently developed further in the followup program SESAR2020. The objective of both research programs was to specify, implement and standardize a modern aeronautical data link that can cope with aviation needs in the long-term. For this purpose, an LDACS specification was produced and LDACS demonstrators were developed to verify the conformity of the implemented LDACS prototypes with the specification. In addition, LDACS standardization in ICAO (International Civil Aviation Organization) has started in December 2016 and LDACS SARPs (Standards and Recommended Practices) have already been developed.
The objective of this paper is to provide a report on the results of the technical validations carried-out in the SESAR2020 solution "PJ.14-02-01 Future Communication Infrastructure (FCI) Terrestrial Data Link". The main focus of these validation activities is to increase the maturity of LDACS and to pave the way for the integration of LDACS into the future ATM communication infrastructure.

\section{PROBLEM STATEMENT}

Today, the terrestrial aeronautical data link is deployed on several dedicated channels in the VHF communication band (118-137 MHz) and it uses relatively old data link technologies: Aircraft Communications Addressing and Reporting System (ACARS), or VHF Data Link (VDL) Mode 2.

There is a common understanding within the ATM community that legacy aeronautical mobile data links will not be able to cope with requirements imposed by advanced ATM concepts. Therefore, the Future Radio System (FRS), as a part of the FCI, will comprise new data link technologies (LDACS, AeroMACS, new SATCOM) as enablers for future ATM concepts.

Since World Radio Conference (WRC) 2012, the lower part of the aeronautical L-band (960-1164 MHz), which has been exclusively used for aeronautical navigation and surveillance services, has also become available for Aeronautical Mobile Route Service (AMRS).

LDACS technology aims therefore to provide air/ground data link capacity and performance in the L-Band that goes beyond what can be provided in the VHF band.

LDACS is a cellular broadband terrestrial line-of-sight communication system using Orthogonal Frequency-Division Multiplexing (OFDM). LDACS Ground Station (GS) is the centralized instance controlling air-ground communication with multiple Airborne Stations (AS) within its radio cell. It uses a star-topology and separate radio channels for Forward Link (FL) and Reverse Link (RL) transmissions.

\section{LDACS DEVELOPMENT}

LDACS technology development started in the SESAR P15.02.04 project, based on an initial LDACS specification [2], and is currently continued in the SESAR2020 PJ14 project. LDACS TX prototypes, which were produced in SESAR 
P15.02.04, were validated against [2] with the results provided in [5].

LDACS A/G data link capability is currently being further developed and tested in SESAR2020 Technological Solution PJ.14-02-01, also named "PJ14 WP3". This project aims at reaching Technology Readiness Level 4 (TRL 4; "technology validated in lab") by the end of 2019. PJ14 WP3 activities target improvements and modifications of [2] that already resulted in an update of the LDACS specification [3] and the first document needed for the LDACS standardization, i.e., ICAO draft LDACS SARPS [4]. The project also clarified the frequencies to be used for LDACS: [3] states that Band I (964 $-1010 \mathrm{MHz}$ ) shall be used for deploying the LDACS Reverse Link (RL), while Band II (1110 - $1156 \mathrm{MHz})$ shall be used for LDACS Forward Link (FL).

Within PJ14 WP3, two companies, Frequentis (AT) and Leonardo (IT), implemented prototype equipment based on requirements provided in [3]. Recently, these prototypes have been validated to demonstrate the progress of LDACS maturity.

Frequentis had already implemented an earlier prototype, however, because of the new specified frequency ranges and the modified transmitter (TX) spectral mask Frequentis had to redesign the LDACS prototypes (Fig.1). In addition to the TX prototype. Frequentis designed and implemented an LDACS Receiver (RX) prototype. Frequentis TX and RX prototypes used in LDACS conformance tests can be configured to act either as LDACS Ground Station (GS) or Airborne Station (AS). These prototypes implement functionalities of LDACS physical layer, including radio functions.

The LDACS TX prototype (Fig. 2) consists of the following functional units:

- Power Supply

- System-on-Chip (SoC) development board with an ARM Cortex processor (LINUX OS) and an FPGA (LDACS baseband processing)

- Digital to Analogue Converter (DAC) board

- $\quad$ RF front-end board

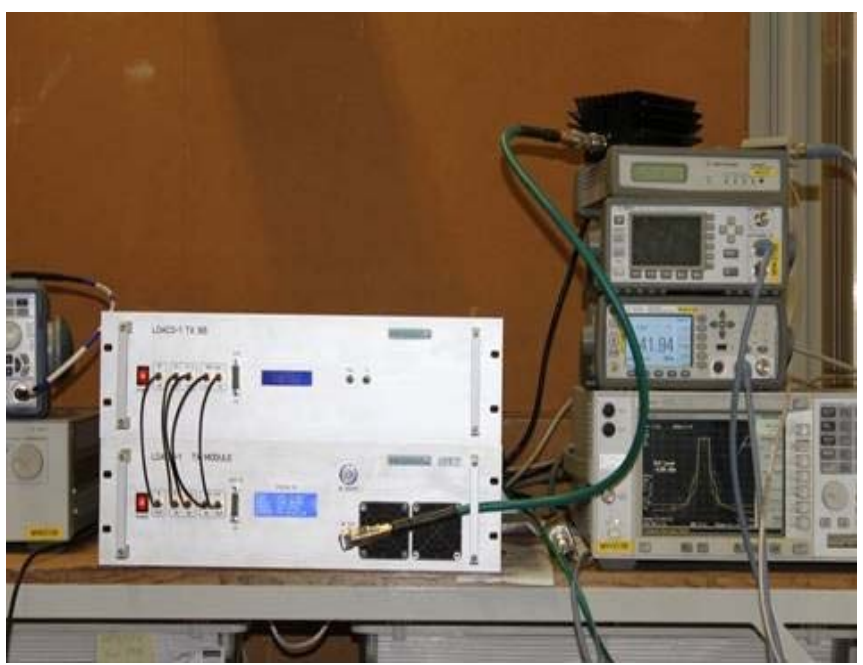

Fig. 1. Laboratory Setup for LDACS Conformance Tests

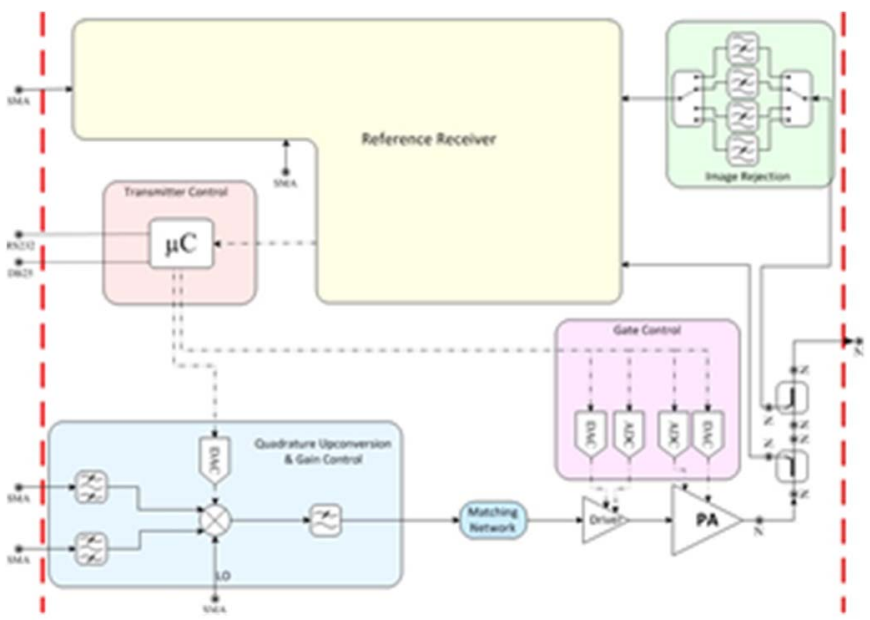

Fig. 2. LDACS TX Block Diagram

The LDACS RX prototype (Fig. 3) comprises the following functional units:

- $\quad$ Power Supply

- System-on-Chip (SoC) development board with an ARM Cortex processor (LINUX OS) and an FPGA (LDACS baseband processing)

- Analogue to Digital Converter (ADC) board

- $\quad$ RF front-end board

Components needed for the LDACS handover tests and which are related to TX/RX layer 2 functionalities - Sub-Network Protocol (SNP), Link Management Entity (LME), Data Link Services (DLS), Medium Access Control (MAC) - are implemented on separate LINUX PCs.

\section{TEChNICAL VALIDATION EXERCISES}

To demonstrate that the targeted maturity level (TRL4 "technology validated in lab") has been reached, SESAR PJ.14-02-01 planed three different kinds of technical validation activities.

(1) LDACS conformance testing: validates the conformance of the RF front-end and physical layer (transmitter and receiver) with the LDACS specification [3].

(2) LDACS interoperability testing: validates the interoperability of LDACS prototypes manufactured by different vendors.

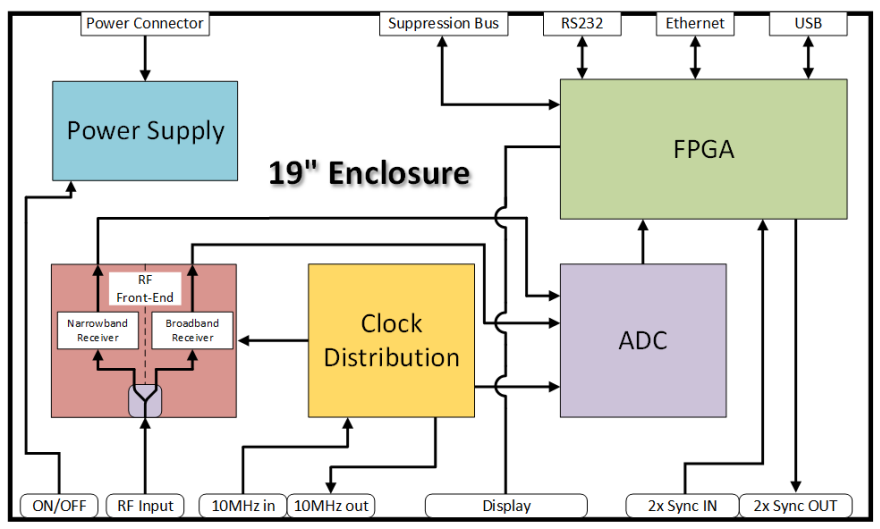

Fig. 3. LDACS RX Block Diagram 
(3) LDACS end-to-end testing: validates the integration of airborne and ground LDACS prototypes within an end-to-end ATM communications infrastructure, as well as mobility-related procedures (information exchanges) when an LDACS airborne station performs a handover from one LDACS ground station to another LDACS GS.

This paper focuses on the results of the conformance testing (1) and the testing of LDACS handover procedures in (3).

\section{LDACS CONFORMANCE TESTS}

\section{A. Verification Approach}

The LDACS conformance tests aimed at verifying if the measured characteristics of the Frequentis LDACS transmitters and receivers comply with the characteristics shown in the specification for the LDACS equipment [3].

Conformance of LDACS transmitters (for GS and AS, respectively) with [3] has been proven by verifying LDACS TX signal via spectrum analyser and oscilloscope.

Conformance of LDACS receivers (for GS and AS, respectively) with [3] has been verified by using a programmable signal generator as source of the LDACS signal. The conclusions about LDACS RX behaviour have been drawn based on the analysis of log files produced during the test campaign.

The schematic view of the LDACS testbed that was used for LDACS conformance tests is shown in Fig.4.

\section{B. Results of LDACS Conformance Tests}

Following aspects of LDACS TX could be successfully tested, both for GS TX and AS TX:

- Operating Power and Frequency Range

- $\quad$ Spectral Mask

- Spectral Flatness

- Broadband Noise

- $\quad$ Spurious Emissions

LDACS TX operating power and frequency ranges have been verified when testing the TX spectral mask, for both GS TX and AS TX operating frequencies.
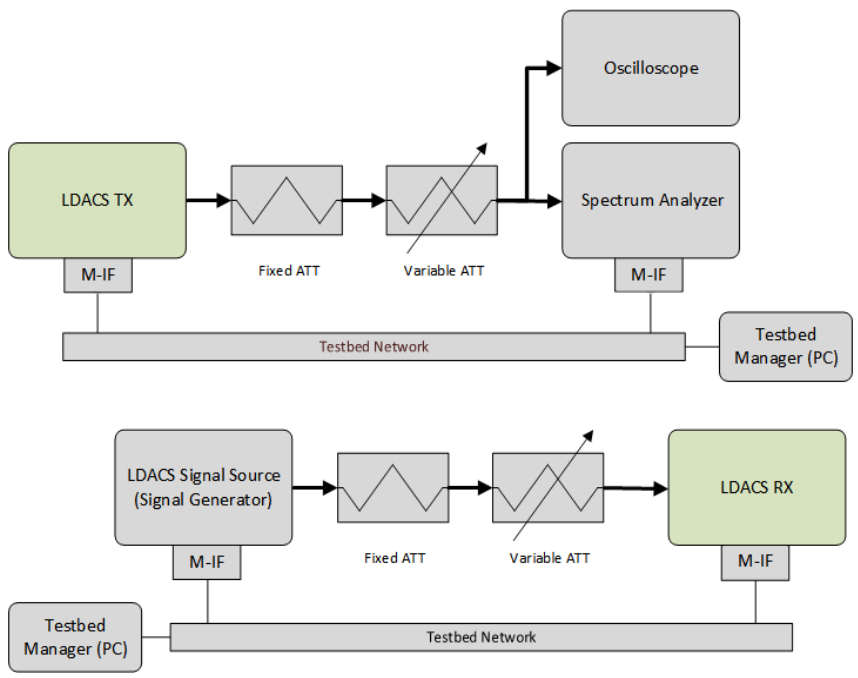

Fig. 4. LDACS Testbed for LDACS Conformance Tests
During these tests, LDACS TX transmitted a continuous sequence of LDACS Super Frames (SF), with an internal SF structure compliant with [3] (including synchronization symbols, null symbols, pilot symbols and data symbols). Data symbols were QPSK modulated, using random data.

Fig. 5 shows the representative LDACS TX spectral mask for the GS TX operating at $1133 \mathrm{MHz}$ with the maximum TX output power $(+42 \mathrm{dBm})$. The blue line shows the measured TX spectral mask, whereas the red line sows the LDACS TX spectral mask from updated LDACS specification [3]. The dotted red line indicates the TX spectral mask according to the previous version of the LDACS specification. It can be seen clearly that the requirements regarding the spectral mask are fulfilled.

Spectral mask conformity tests were successfully performed for other GS TX frequencies (1110 MHz, 1117.5 MHz, $1125 \mathrm{MHz}, 1133 \mathrm{MHz}, 1156 \mathrm{MHz}$ ), as well as for AS TX frequencies (964 MHz, $987 \mathrm{MHz}, 971.5 \mathrm{MHz}, 979 \mathrm{MHz}$, $1010 \mathrm{MHz}$ ).

LDACS TX spectral flatness within the LDACS signal bandwidth was successfully verified for different GS and AS TX operating frequencies and power settings.

LDACS specification [3] contains no explicit requirement for TX broadband noise, thus the achieved TX noise performance of the prototype TX equipment has been measured according to initial LDACS specification [2] to get insight in potential improvements. According to [2], the broadband noise power density shall not exceed $-133 \mathrm{dBc} / \mathrm{Hz}$. With an LDACS power of $+42 \mathrm{dBm}(\sim 0 \mathrm{dBc})$, this value corresponds to a noise power density of $-91 \mathrm{dBm} / \mathrm{Hz}$. Measurements performed with the GS TX and AS TX showed (Fig. 6) that the achieved phase noise floor is far below the limit specified in the [2] requirements.

TX spurious performance is not specified in [3] either, but the spurious frequencies and levels for the prototype TX equipment have been recorded to get an indication about requirements for the AS duplexer, as well as for the bandpass filter following the GS TX.

Following aspects of LDACS RX were successfully tested:

- Frequency Range

- Sensitivity When Receiving Data Frames

- Reception at Maximum LDACS Signal Level

- Adjacent Channel Blocking Performance

These tests have been performed with the AS RX. Note that the same physical LDACS RX unit can be configured to act as either GS RX or AS RX. RX ability to operate on various frequencies within its operating frequency range has been verified when testing the RX sensitivity. Sensitivity was measured for three operating frequencies $(1110 \mathrm{MHz}, 1111.5 \mathrm{MHz}$, $1125 \mathrm{MHz}$ ). The worst-case obtained sensitivity value for these frequencies was $-107 \mathrm{dBm}$.

RX sensitivity, when receiving FL data frames, is defined as LDACS signal power at RX input for which $\mathrm{BER}=10^{-6}$ is achieved.

Fig. 7 shows the BER versus average LDACS signal power at RX input at $1110 \mathrm{MHz}$ and $1125 \mathrm{MHz}$, respectively. 


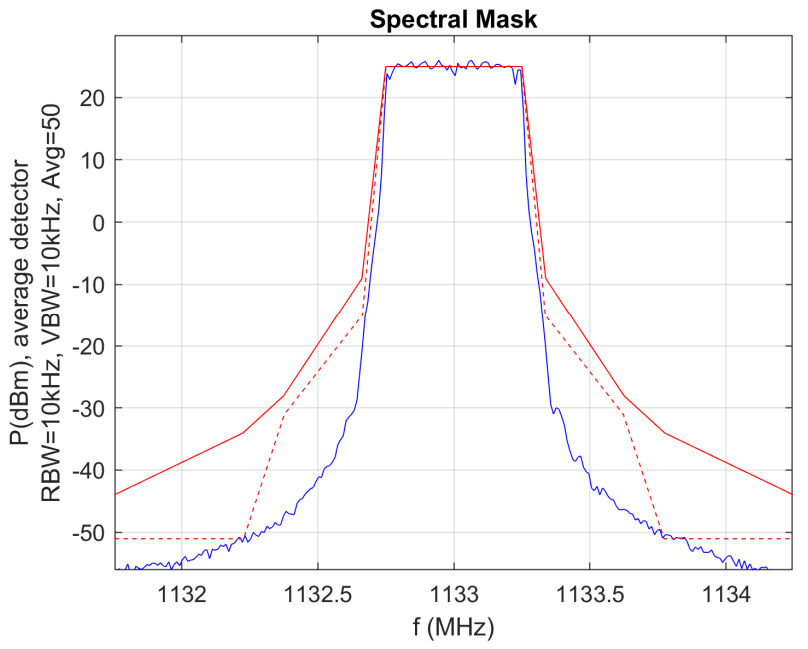

Fig. 5. LDACS TX Spectral Mask

LDACS RX ability to process and decode FL data frames received with specified [3] peak signal power $(-10 \mathrm{dBm})$ has been verified for frequencies $1110 \mathrm{MHz}, 1117.5 \mathrm{MHz}$ and $1125 \mathrm{MHz}$.

According to [3], LDACS TX Peak to Average Power Ratio (PAPR) shall not exceed $11 \mathrm{~dB}$. Therefore, peak signal power of $-10 \mathrm{dBm}$ translates into an average signal power of $21 \mathrm{dBm}$. The tests have been performed with $-20 \mathrm{dBm}$ LDACS average signal power at RX input, which is $1 \mathrm{~dB}$ above the required value.

The test payload size used for the BER tests was sufficient to reliably detect bit errors at BER $=10^{-6}$ or below. However; during these tests no bit errors were observed $(\mathrm{BER}=0)$, which confirms that the LDACS RX can operate at prescribed peak input signal power level.

The LDACS specification [3] contains no explicit requirement for adjacent channel blocking performance, however, the blocking performance has been tested, to get an indication about possible impact of a strong CW signal operating on adjacent LDACS channel. Adjacent channel blocking performance has been checked for single operating frequency (1111 $\mathrm{MHz}$ ).

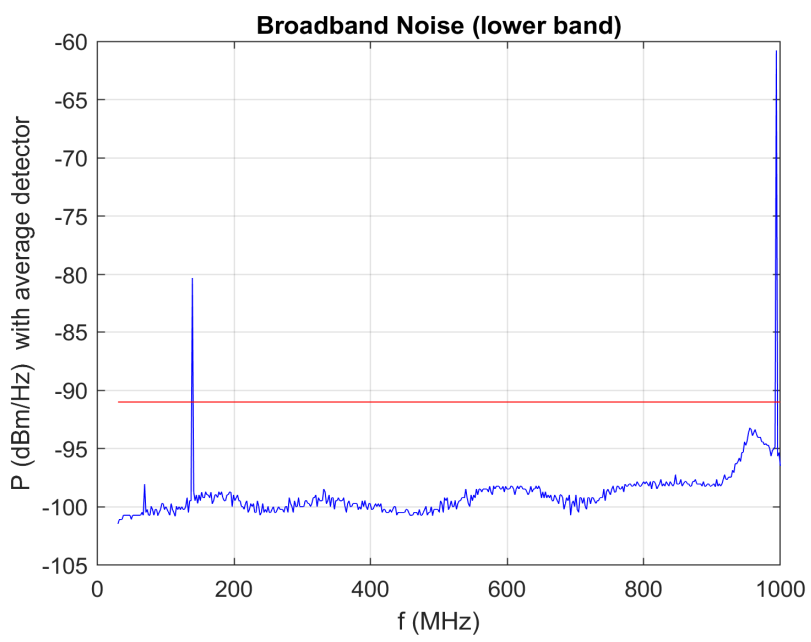

Fig. 6. LDACS TX Broadband Noise

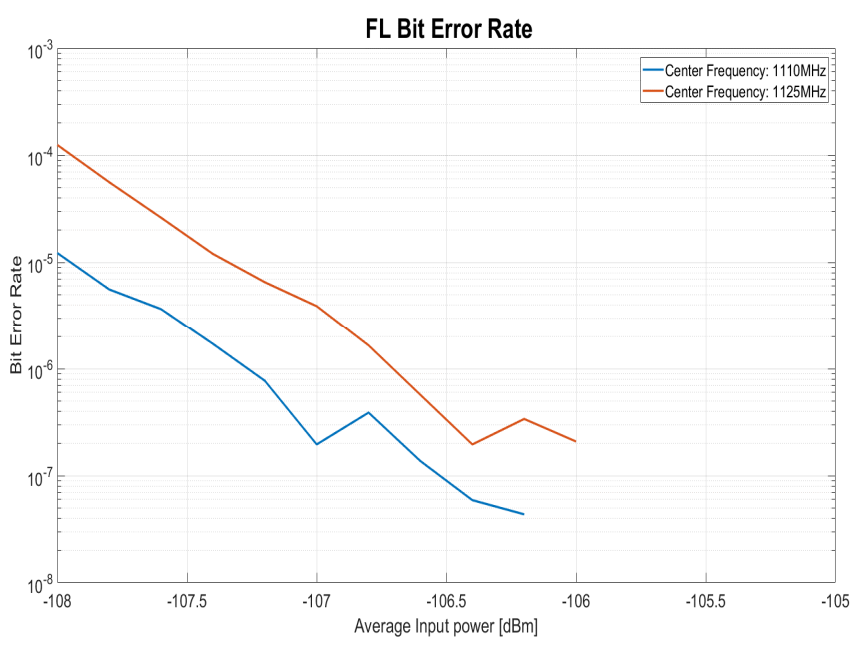

Fig. 7. LDACS RX Sensitivity

LDACS signal with average power $3 \mathrm{~dB}$ above the RX sensitivity $(-101 \mathrm{dBm})$ was present at the input of the LDACS receiver. Then, a CW signal on the upper and lower adjacent channel $( \pm 0.5 \mathrm{MHz}$ offset from LDACS RX operating frequency) was activated and the CW signal level successively increased.

With sufficiently large test payload (sufficient number of LDACS super-frames for detecting BER $=10^{-6}$ or below) the measured BER was zero when the $\mathrm{CW}$ signal level was $70 \mathrm{~dB}$ above the LDACS signal level.

\section{LDACS HANDOVER TESTS}

\section{A. Verification Approach}

The testbed used for handover (HO) tests is shown in Fig. 8. The numbers shown in Fig. 8 refer to internal functional entities e.g., A-ES (8) denotes the Airborne End System.

Note: The LDACS architecture foresees an airborne router $(A-R)$ in the aircraft. However; $A-R$ is not required for the execution of the handover tests, therefore it was not implemented in the validation platform.

The testbed comprises one AS (7) and two ground-stations, GS1 (4) and GS2 (5). A single physical PC - VM host (1) - has been used for implementing several functions: Testbed Manager (TBM), Access Router (AC-R), and Ground End System (G-ES). TBM (1c) within the VM host is connected to the Management LAN and can access all other components of the testbed. TBM and all other entities use IP4 stack for communication over the Management LAN.

AS, GS1 and GS2 implement layer 2 interfaces over which these entities communicate with external network entities (AES, AC-R). AC-R (1b) uses the IPv6 stack of the VM host (1).G-ES (1a) is implemented in a virtual machine (VM) within the VM host and uses its own IPv6 stack. As VM host (1) provides only one Ethernet interface, two separate pointto-point connections between two internal AC-R interfaces and GS1/GS2, respectively, have been realized by using VLAN technology, supported by the Managed Switch (MSW, 3).

LDACS Medium Access Control (MAC) entities in AS and GSs are interconnected over a Software-Defined Network (SDN) switch (6), instead of using physical LDACS PHY and TX/RX components. 
Note: The handover test connects the LDACS layer 2 implementations directly without using the physical layer already validated above.

Prior to handover, SDN-SW is configured (from AS) such that AS is connected to GS1. At a certain point in time, SDN$\mathrm{SW}$ is programmatically manipulated (from AS) such that AS will become connected to GS2 (previous connection to GS1 breaks). This emulates frequency switchover between the involved GSs. At roughly the same time the AC-R routing table is programmatically modified such that AC-R will from now onwards send Forward Link (FL) IP packets to GS2 rather than to GS1. This emulates the micro-mobility handover without having to implement any specific mobility solution within the handover testbed.

The selected approach allowed to focus on verifying internal LDACS mechanisms during $\mathrm{HO}$ without having to consider the additional impact of mobility-related signalling in the ground network.

\section{B. Results of Handover Tests}

When LDACS AS moves between LDACS GSs, the exchange of data between layer 3 entities (hosts, routers), interconnected via LDACS data link, should continue without loss of data.

This requires the proper implementation of LDACSinternal mechanisms, including exchanges of LDACS controlplane messages between AS and GS(s) within LDACS testbed. Furthermore, involved layer 2 entities - Sub-Network Protocol (SNP), Link Management Entity (LME), Data Link Services (DLS), Medium Access Control (MAC) - must operate according to the descriptions provided in [3].

Prior to handover, it must be shown that A-ES and G-ES hosts can bidirectionally communicate over AS-GS1 LDACS link. After AS performed HO from GS1 to GS2 end-to-end communications should be re-established over AS-GS2 link.

Following specific atomic test cases have been investigated within the handover tests:

(1) AS Cell Entry at GS1

(2) RL Resource Allocation at GS1

(3) FL Resource Allocation at GS1

(4) AS Preparing HO and Cell Exit at GS1

(5) AS Cell Entry at GS2

(6) RL Resource Allocation at GS2

(7) FL Resource Allocation at GS2

Prior to executing any test case it was verified that the LDACS testbed is properly configured. Then, the above sequence of test cases has been executed.

It is important to note that above control-plane procedures run in parallel with data-plane exchanges. For the purposes of the LDACS handover tests, data-plane traffic has been simulated by using the ICMPv6 ping facility that is supported by any IPv6 host or router.

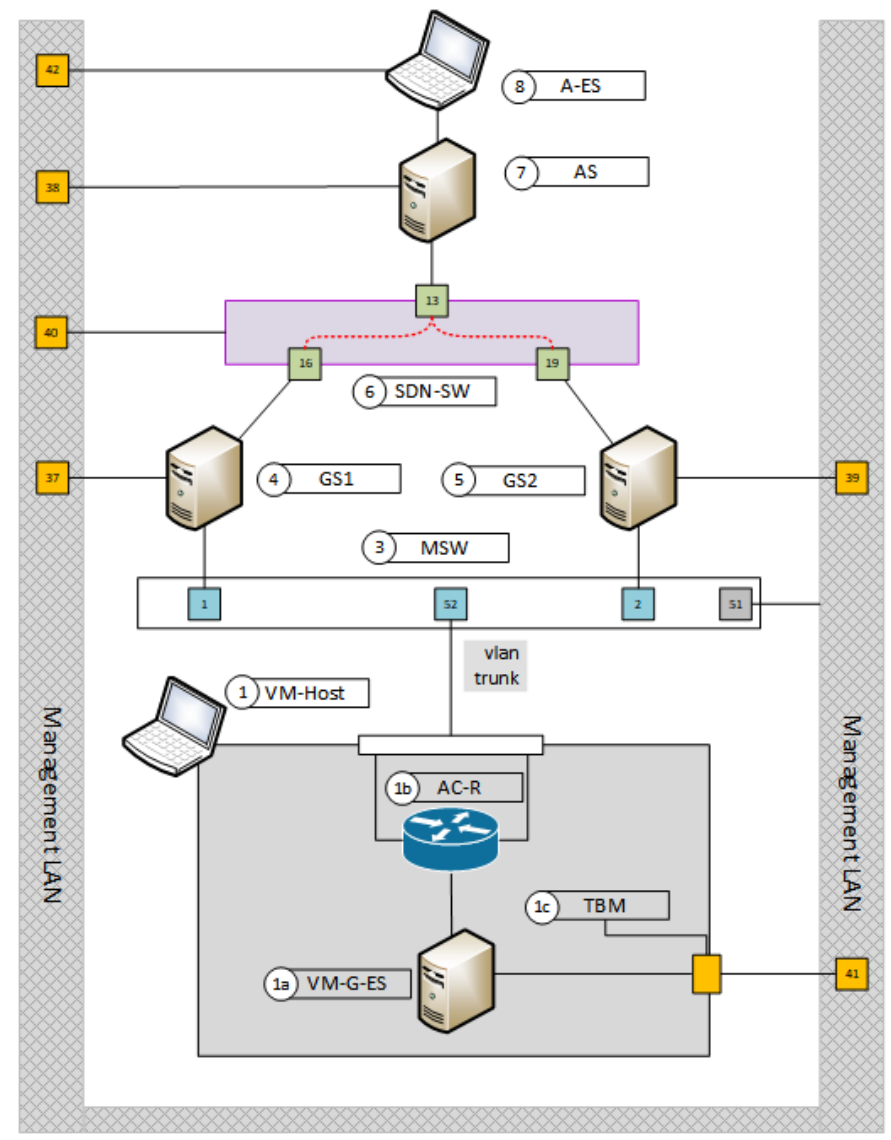

Fig. 8. LDACS Testbed for Handover Tests

After an initial AS attachment to GS1 the A-ES host starts sending Ping Echo Requests to the peer G-ES host that responds by sending back Ping Replies.

After being activated, AS, GS1 and GS2 start producing entries in their associated log files and continue across all test cases until they are switched off. To make the post-measurement analysis easier, time-stamped entries from three separate $\log$ files have been merged into single log file. Representative events related to above test cases and associate procedures could be identified by analysing entries in the combined log file.

Wireshark capturing is enabled at A-ES and G-ES interfaces. These PCAP files have been converted into plain text files. Changes in the connectivity status between AS and GS1/GS2, respectively, could be identified by inspecting these files.

In the LDACS handover tests, it could be successfully demonstrated that LDACS layer 2 entities implemented in the AS and the GS could exchange control-plane signalling messages - as required for the concerned procedures - over the emulated air interface. The procedures described in [3] have been visualized by using UML time-sequence diagrams.

It could be demonstrated that procedures related to the AS cell entry at certain GSs (either initial AS attachment or reattachment after the $\mathrm{HO}$ ), as implemented within prototype LDACS AS and GS equipment, are conform with the behaviour described in [3]. Furthermore, it could be verified that all involved layer 2 entities - SNP, LME, DLS, MAC - operate as specified. 
Similarly, proper behaviour of LDACS layer 2 entities could be demonstrated for other procedures (FL/RL resource allocation, HO preparation, Cell exit).

FL and RL resource allocation procedures in the control plane run in parallel to data exchanges in the user plane. Ping Echo Requests and Ping Replies are internally buffered within LDACS AS and GS during FL/RL allocation procedures, so no packets are dropped as long as the connection between AS and GS remains stable.

Within the LDACS handover tests, it was also shown that prototype LDACS AS, which was initially connected to GS1, could perform HO to and establish connection with GS2 that uses different FL/RL channels than GS1 with acceptable impact on ongoing end-to-end communications. The typical measured HO duration was $1.5 \mathrm{~s}$, comprising the delay due to LDACS FL/RL framing constraints, time required for internal processing of control-plane messages in AS/GS entities and also time required for manipulating AC-R routing table using its command line interface.

\section{FUTURE WORK}

SESAR2020 project "PJ.14-02-01 FCI Terrestrial Data Link" developed LDACS prototypes to validate the air interface requirements and to support the validation of ATN IPS infrastructure. In addition, the project has defined the LDACS architecture and it continuously supports the LDACS standardization that has already been started in the context of ICAO.

While significant progress is expected to be achieved in the context of PJ.14-02-01, further work will be required, particularly for assuring LDACS spectrum compatibility with other L-band systems and supporting validation and standardization. The SESAR2020 program plans that current activities (wave 1) will be completed by end of 2019 and new activities (wave 2) will be defined to continue the required work (2020 and beyond).

LDACS conformance tests have demonstrated that physical layer requirements and radio requirements according to [3] have been properly implemented in prototype LDACS TX and $\mathrm{RX}$ equipment produced by Frequentis. These tests demonstrated the technical feasibility of the current approach. Important performance parameters have been verified in an interference-free environment.

However, further testing will be required to check the remaining [3] detailed aspects that could not be considered in this testing campaign. Such aspects comprise e.g. A/A data link functionality, digital $\mathrm{A} / \mathrm{G}$ voice functionality, A-PNT (Alternative Positioning Navigation and Timing) functionality and all aspects related to security.

Operation of LDACS together with other L-band systems, considering mutual interference, shall be also tested in followon activities.
HO trials were conducted by using a Software-Defined Network (SDN) switch for emulating AS frequency switchover between involved GSs, instead of using TX/RX hardware.

LDACS AS LME and MAC state machines were not implemented in the prototype LDACS equipment and were not tested and will have to be validated in future activities.

Further testing will also be required after physical TX/RX components become fully integrated within the prototype equipment. This also includes the aspects related to Automatic Gain Control (AGC) and methods for scanning adjacent LDACS ground stations, inclusive handling of associated MAC messages.

Additional testing may also be required when the end-toend ATN/IPS solutions for managing mobility and multilink become selected.

\section{CONCLUSION}

This paper provides a status report on the current progress of LDACS validation within SESAR2020, regarding the verification of LDACS radio conformance and LDACS handover procedures.

LDACS conformance tests have demonstrated correct implementation of LDACS physical layer and radio front-end functionalities in the LDACS TX and RX equipment produced by Frequentis.

LDACS handover tests demonstrated the correct implementation of the LDACS layer 2 user plane, as well as the correct implementation of the LDACS layer 2 control plane functions supporting mobility management. Additionally, the duration of the handover between LDACS ground stations has been measured.

The LDACS verification and validation activities show clearly that LDACS can be implemented with the expected performance results and indicate the next steps for increasing the technology readiness.

\section{ACKNOWLEDGMENT}

This project received funding from the SESAR Joint Undertaking under the Grant Agreement No 734168 under the European Union's Horizon 2020 research and innovation program. The views expressed in this paper are those of the authors.

\section{REFERENCES}

[1] SESAR European ATM Master Plan Executive view, 2015. Available at: https://www.atmmasterplan.eu

[2] M. Sajatovic, M. Schnell, "Updated LDACS1 Prototype Specification”, Ed 00.00.02, SJU Project 15.02.04, EWA04-1-T2-D2, 2010

[3] T. Gräupl, C. Rihacek, B. Haindl, Q. Parrod, "LDACS A/G Specification”, Ed. 00.01.01, SESAR.IR-VLD.Wave1-17-2015 CNS, 2018

[4] Draft LDACS SARPS, ICAO, SECOND MEETING OF THE DATA COMMUNICATION INFRASTRUCTURE WORKING GROUP (DCIWG), Montreal, Canada, 22 to 26 October 2018

[5] B. Haindl, J. Meser, M. Sajatovic, S. Müller, H. Arthaber, T. Faseth, M. Zaisberger, "LDACS Conformance and Compatibility Assessment", DASC 2014 